\title{
La amiodarona es más efectiva que otros antiarrítmicos para prevenir la recurrencia de la fibrilación auricular
}

Amiodarone to prevent recurrence of atrial fibrilation.

Roy D, Talajio M, Dorian P. N Engl J Med 2000; 342:913-20

\section{Objetivo}

Evaluar si el tratamiento con amiodarona en dosis bajas es más eficaz en la prevención de recurrencias de la fibrilación auricular (FA) que la terapia antiarrítmica con sotalol o propafenona.

\section{Diseño}

Ensayo clínico multicéntrico, prospectivo, aleatorizado no ciego con un seguimiento promedio de un año y medio.

\section{Lugar}

Estudio multicéntrico realizado en 19 centros cardiológicos de Canadá.

\section{Pacientes}

Pacientes que hayan tenido un episodio de FA de al menos 10 minutos de duración en los seis meses precedentes a la aleatorización.

\section{Intervención}

De los 403 pacientes incluidos, 201 se aleatorizaron para recibir amiodarona $(10 \mathrm{mg} / \mathrm{kg}$ día por 14 días seguido de $300 \mathrm{mg} /$ día por 4 semanas y luego $200 \mathrm{mg} /$ día) y un segundo grupo se aleatorizó para recibir sotalol $160-320 \mathrm{mg} /$ día en dos tomas o propafenona 450-600 mg/día en dos tomas (ajustado por edad, peso corporal y función renal). Cuando alguna de estas 2 drogas fallaba se probaba con la otra. Si a los 21 días de la aleatorización no se alcanzaba el ritmo sinusal se realizaba cardioversión eléctrica a pacientes de cualquier grupo y se los clasificaba como recurrencia.

\section{Resultados principales}

La amiodarona redujo significativamente la tasa de recurrencia, tanto de FA paroxística como persistente (RRR* de $57 \%$ [IC $95 \% 43-68 \%$ ]) y RRA* del $28 \%$. El numero de pacientes necesario para tratar (NNT) fue cuatro, es decir que sólo necesito tratar cuatro pacientes durante 16 meses con Amiodarona en lugar de sotaloL o propafenona para prevenir una recurrencia de FA. En dicho período de un seguimiento el $35 \%$ de los pacientes asignados a amiodarona y el $63 \%$ de los pacientes asignados al segundo grupo tuvieron su primera recurrencia de FA ( $p 0,001)$.

La tasa de recùrrencias fue virtualmente la misma para los asignados al sotalol o la propafenona. El $18 \%$ de los pacientes asignados a amiodarona y $11 \%$ de los pacientes asignados al segundo grupo discontinuaron el estudio por efectos adversos.

\section{Conclusiones}

El tratamiento con amiodarona demostró ser más eficaz que el sotalol o la propafenona en la reducción de recurrencia de FA.

\section{COMENTARIO}

La tendencia más reciente en el manejo de la FA es restaurar el ritmo sinusal siempre que sea posible con el fin de controlar los síntomas, mejorar la capacidad funcional y reducir el riesgo de embolismo. ${ }^{1}$ Sin embargo la FA recurre en al menos la mitad de los pacientes tratados en un lapso de 3-6 meses.2, 3 El tratamiento a largo plazo con quinidina y otros antiarrítmicos clase I ha sido cuestionado. Esto se debe a que análisis retrospectivos, ${ }^{*}$ con un alto porcentaje de pacientes con cardiopatía orgánica, sugirieron que estos agentes aumentarían la mortalidad a dos años respecto a los controles (1.6$2.3 \%$ vs. $0.68 \%$ respectivamente). 2,4 Por el contrario, la amiodarona tiene un mínimo efecto proarrítmico (capacidad de generar nuevas arritmias) y no parece aumentar la mortalidad. 5, 6 En el estudio que analizamos se verifica, además, que la amiodarona tiene el doble de eficacia que el sotalol o la propafenona en la prevención de recurrencia de FA durante un seguimiento medio de 1.3 años. Esto se observó en todos los subgrupos considerados y es consistente con los siete estudios no controlados publicados previamente, que mostraron una eficacia promedio del $71 \%$ en mantener el RS a largo plazo. ${ }^{7,8} \mathrm{~A}$ pesar de no tratarse de un estudio doble ciego el resultado primario fue fácilmente objetivable y evaluado por un comité que desconocía el tratamiento asignado. Sin embargo, un diseño abierto está sujeto al sesgo de una búsqueda más exhaustiva de efectos adversos, especialmente con una droga de mala reputación como la amiodarona. Esto último se debe a las altas dosis acumulativas en que fue utilizada en EE.UU. para prevenir arritmias ventriculares refractarias a antiarrítmicos clásicos. A pesar de esto, la amiodarona fue bien tolerada y los eventos adversos aceptables. Un 18\% abandonó la amiodarona por efectos adversos, cifra inferior a la reportada en grandes estudios. 5, 6 Debe destacarse que los efectos adversos a muy largo plazo no han sido totalmente estudiados (los seguimientos de los mayores ensayos no suelen pasar los dos años) aunque en la Argentina existe una extendida experiencia con el uso de este fármaco. Es interesante señalar que el GESICA (Grupo Argentino de la Sobrevida en la IC en la Argentina) demostró que en pacientes con insuficiencia cardíaca con disfunción sistólica, la amiodarona mejora la sobrevida, conclusión corroborada en meta-analisis ${ }^{5,6}$ que mostraron un $13 \%$ de reducción global de la mortalidad total en pacientes de alto riesgo post-IAM reciente o con insuficiencia cardíaca. Por su mayor seguridad y efectividad la amiodarona parece ser la droga de elección para el mantenimiento del RS, especialmente en pacientes con cardiopatía orgánica de base.1,9

*Ver glosario

\section{Dr. Carlos Cimino}

Dr. Agustín Ciapponi

Unidad de Medicina Familiar y Preventiva. Hospital Italiano de Buenos Aires.

\section{Referencias}

1. Reiffel JA. Impact of structural heart disease on the selection of class III antiarrhythmics for the prevention of atrial fibrillation and flutter. Am Heart J 1998; 135:551-

2. Coplen SE, Efficacy and Safety of quinidine therapy for maintenance o sinus rhythm after cardioversión. Circulation 1990, 82:1106-16 [erratum, Circulation 1991;83:714]

3. Nattel,S. The treatment of atrial fibrillation:an evaluation of drug therapy, electrical modalities and therapeutic considerations. Drugs 1994; 48:345-71.

4. Flaker GC. Antiarrhythmic drug therapy and cardiac mortality en atrial fibrilation. J Am Coll Cariol 1992;20:527-532.

5. Vorperian VR. Adverse effects of low dose amiodarone: a meta-analysis. J Am Coll Cardiol 1997: 30:791-8.

6. Amiodarone Trials Meta-Analysis Investigators. Writing committee: Connolly S., Cairns J., Gent M., Roberts R, Yusuf S. Effect of prophylactic amiodarone on mortality after acute myocardial infarc-

tion and in congestive heart failure: meta-analysis of individual data from 6500 patients en randomised trials. Lancet 1997; 350:1417-24

7. Nattel S. Newer Developments in the Management of Atrial Fibrillation. Am Heart J 1995 130:1094-106

8. Podrid PJ. Amiodarona Reevaluation of an Old Drug. Ann Intern Med 1995 122; 689-700

9. Pratt CM, Waldo AL. A symposium: treatment of Atrial Fibrillation in the Era of Managed Care. Am J Cardiol. 1998 Mar 12; 81(5A): 1C-45C. 